\title{
Breast cancer outcome in relation to bone mineral density and bisphosphonate use: a sub-study of the DATA trial
}

\author{
Irene E. G. van Hellemond ${ }^{1}$ - Carolien H. Smorenburg ${ }^{2}$ • Petronella G. M. Peer ${ }^{3}$ - Astrid C. P. Swinkels ${ }^{4}$. \\ Caroline M. Seynaeve ${ }^{5}$. Maurice J. C. van der Sangen ${ }^{6}$. Judith R. Kroep ${ }^{7}$. Hiltje de Graaf ${ }^{8}$. Aafke H. Honkoop ${ }^{9}$. \\ Frans L. G. Erdkamp ${ }^{10}$. Franchette W. P. J. van den Berkmortel ${ }^{11}$. Wilfred K. de Roos ${ }^{12}$. Sabine C. Linn ${ }^{13}$. \\ Alexander L. T. Imholz ${ }^{14}$. Maaike de Boer ${ }^{1} \cdot$ Vivianne C. G. Tjan-Heijnen ${ }^{1,15}$ on behalf of the Dutch Breast Cancer \\ Research Group (BOOG)
}

Received: 29 October 2019 / Accepted: 3 February 2020 / Published online: 2 March 2020

(c) The Author(s) 2020

\begin{abstract}
Purpose The phase III DATA study compared 6 and 3 years of adjuvant anastrozole following 2-3 years of tamoxifen in postmenopausal breast cancer patients. This pre-planned side-study assessed the relationship between a reduced bone mineral density (BMD) and distant recurrence-free survival (DRFS), and evaluated the effect of bisphosphonates on DRFS.

Methods We selected all patients with a BMD measurement within 3 years after randomisation (landmark) without any DRFS events. Kaplan-Meier methods and Cox proportional hazards models were used for analyses.

Results Of 1860 eligible patients, 1142 had a DEXA scan before the landmark. The BMD was normal in $436(38.2 \%)$ and showed osteopenia in 565 (49.5\%) and osteoporosis in 141 (12.3\%) patients. After a median follow-up of 5.0 years from the landmark, neither osteopenia nor osteoporosis (compared with normal BMD) were associated with DRFS in both the 6-year [osteopenia HR 0.82 (95\% CI 0.45-1.49), osteoporosis HR 1.10 (95\% CI 0.26-4.67)] and the 3-year arm [osteopenia HR 0.75 (95\% CI 0.40-1.42), osteoporosis HR 1.86 (95\% CI 0.43-8.01)]. Moreover, bisphosphonate use did not impact DRFS. Conclusion No association was observed between a reduced BMD and DRFS. Neither did we observe an impact of bisphosphonates on DRFS.
\end{abstract}

Keywords Breast cancer · Tamoxifen - Aromatase inhibitor · Bone health $\cdot$ Osteoporosis $\cdot$ Bisphosphonates $\cdot$ Survival . Bone metastases $\cdot$ Distant recurrence-free survival

\section{Introduction}

Bisphosphonates, in addition to supplementation of vitamin D and calcium, are pivotal in the medical treatment of osteoporosis. Aside from preventing bone loss and fractures, the use of bisphosphonates in the adjuvant setting has been

Part of these results were presented at the poster session of the 2018 San Antonio Breast Cancer Symposium, abstract number 810.

Electronic supplementary material The online version of this article (https://doi.org/10.1007/s10549-020-05567-9) contains supplementary material, which is available to authorized users.

Vivianne C. G. Tjan-Heijnen

vcg.tjan.heijnen@mumc.nl

Extended author information available on the last page of the article shown to improve breast cancer outcome in postmenopausal breast cancer patients [1-6]. The Early Breast Cancer Trialists' Collaborative Group (EBCTCG) meta-analysis observed a significant improvement in the rate of distant recurrences $(18.4 \%$ in the bisphosphonate group versus $21.9 \%$ in the group without bisphosphonate, $p<0.001$ ), mainly driven by a reduction in bone recurrences $(5.9 \%$ versus $8.8 \%$, respectively, $p<0.001$ ), and a lower 10 -year breast cancer mortality [7]. The effect was seen irrespective of bisphosphonate type. Additionally, in epidemiological studies bisphosphonate use for osteoporosis in healthy postmenopausal women was associated with a $30 \%$ reduced risk of breast and colon cancer [8]. Moreover, neoadjuvant use of bisphosphonates in combination with chemotherapy in women with stage II/III breast cancer resulted in an improved clinical and pathological response rate in postmenopausal women only [9]. Nevertheless, it remains 
insufficiently clear how to explain the effects of bisphosphonates on breast cancer prevention and recurrence, respectively. Is the effect directly caused by the bisphosphonates? Or are women with early breast cancer and osteoporosis simply at a lower risk of developing metastases due to lower oestrogen levels than in those without osteoporosis? Also, earlier studies showed that a reduced bone mineral density (BMD) was associated with a lower risk of breast cancer $[10,11]$.

The phase III DATA trial investigated the efficacy of 6 versus 3 years of anastrozole after an initial 2-3 years of tamoxifen in postmenopausal women with early breast cancer. Earlier, we reported on patterns of care considering bone health in these women, and the trend of BMD over time, and the incidence of fractures during and after cessation of anastrozole treatment [12]. In the current pre-planned sidestudy we assessed the relationship between a reduced BMD and late distant recurrence-free survival (DRFS) (more than 5 years after breast cancer diagnosis), and evaluated the effect of bisphosphonates on late DRFS.

\section{Methods}

\section{Study design, participants and procedures}

The DATA trial included 1860 eligible postmenopausal women with hormone receptor-positive early breast cancer who had already received 2-3 years of adjuvant tamoxifen after curative local treatment, and who were without signs of loco-regional and/or distant metastases. Patients used anastrozole for 6 or 3 years according to randomisation. Ethics approval was obtained at the central commission of research involving humans in Nijmegen in the Netherlands. The DATA trial (NCT00301457) is described in detail elsewhere [13]. Decisions on BMD measurements and bisphosphonate use were left to the treating physician. The DATA study protocol advised to follow the recommendations of (inter)national guidelines. During the conduct of the study adjuvant bisphosphonates were not recommended, therefore they were predominantly prescribed as treatment for osteopenia and osteoporosis ( $T$-score $\leqq-2.0)$. We registered all BMD measurements and start of bisphosphonate use. BMD was measured by a dual-energy $\mathrm{x}$-ray absorptiometry (DEXA) scan of the lumbar spine/hip. For the current analyses, DATA patients were selected who had a DEXA scan within 3 years after randomisation and did not have any distant recurrences or death (flow chart shown in Supplemental Fig. 1). Of the 1860 patients who were eligible for the DATA trial, 1714 patients had not experienced a DRFS event before the 3-year landmark. Amongst them, 1142 had at least one BMD measurement before the 3-year landmark.

\section{Statistical analysis}

We registered all results of DEXA scans performed within three years after randomisation. The outcomes ( $T$-scores) were categorised according to the world health organization classification for BMD; normal BMD $T$-score $\geq-1.0$ standard deviation (SD), osteopenia $T$-score $<-1.0$ and $>-2.5$ $\mathrm{SD}$, and osteoporosis $T$-score $\leq-2.5 \mathrm{SD}$ [14]. Assessment of osteopenia and osteoporosis was based on the lowest available $T$-score in either the hip or the lumbar spine. Based on the result of the DEXA scan we classified the patients in three groups (normal BMD, osteopenia, and osteoporosis). The landmark method was used to assess the survival after a particular point in time. The DRFS time was measured from the landmark of 3 years after randomisation to distant recurrence or death, the so-called residual survival, and was censored at the date of last follow-up. DRFS rates were obtained with the Kaplan-Meier method. We analysed the relationship between BMD and DRFS by comparing women having either osteopenia or osteoporosis with those having a normal BMD in a Cox proportional hazards model. Secondly, we performed the same analyses selecting only those patients who had not received bisphosphonates before the landmark of 3 years, thereby correcting for a potential effect of bisphosphonates on DRFS. The hazard ratios (HR) were adjusted for tumour status, nodal status, tumour grade, and hormone receptor status. Further, we evaluated the effect of bisphosphonates, started before the 3-year landmark for a reduced BMD, on late DRFS by comparing the women with and without bisphosphonates. We used the term late DRFS since these recurrences occurred more than 5 years after breast cancer diagnosis. All reported $P$ values are two-sided and a $p$ value $\leq 0.05$ was considered statistically significant. All analyses were performed using SAS version 9.2.

\section{Results}

Of the 1860 randomised eligible DATA patients, 1142 (65.5\% in the 6-year arm and 62.9\% in the 3-year arm) had a DEXA scan within the first 3 years after randomisation. The median age at randomisation was 57.5 years (interquartile range $51.0-63.0), 67.3 \%$ of the patients had node-positive disease and $71.3 \%$ underwent (neo-)adjuvant chemotherapy. Except for T-stage, the baseline characteristics were well balanced between the BMD groups (Table 1). Women with a normal BMD more frequently had a larger tumour size at diagnosis, but the patient characteristics were similar to these of the total study population (Supplemental Table 1).

At the 3-year landmark, the BMD was considered normal in $436(38.2 \%)$, showed osteopenia in $565(49.5 \%)$, 
Table 1 Baseline characteristics of all eligible randomised patients in the DATA study who underwent a DEXA scan before the landmark of 3 years after randomisation

\begin{tabular}{|c|c|c|c|c|}
\hline Characteristic & Total group $(n=1142)$ & Normal BMD $(n=436)$ & Osteopenia $(n=565)$ & Osteoporosis $(n=141)$ \\
\hline \multicolumn{5}{|l|}{ Age at randomisation-no. (\%) } \\
\hline Median age at randomisation (IQR) & $57.5(51.0-63.0)$ & $56.8(51.0-62.0)$ & $57.6(51.0-64.0)$ & $59.0(51.0-64.0)$ \\
\hline$<49$ years & $227(19.9)$ & $94(21.6)$ & $113(20.0)$ & $20(14.8)$ \\
\hline $50-59$ years & $462(40.5)$ & $175(40.1)$ & $227(40.2)$ & $60(42.6)$ \\
\hline$\geq 60$ years & $453(39.7)$ & $167(38.3)$ & $225(39.8)$ & $61(43.3)$ \\
\hline \multicolumn{5}{|l|}{ Tumour status-no. (\%) } \\
\hline pT1 & $519(45.5)$ & $170(39.0)$ & $276(48.9)$ & $73(51.8)$ \\
\hline pT2 & $540(47.3)$ & $237(54.4)$ & $244(43.3)$ & $59(41.8)$ \\
\hline pT3/4 & $82(7.2)$ & $29(6.7)$ & $44(7.8)$ & $9(6.4)$ \\
\hline Unknown & 1 & 0 & 1 & 0 \\
\hline \multicolumn{5}{|l|}{ Nodal status-no. $(\%)$} \\
\hline $\mathrm{pN} 0 / \mathrm{pN} 0(\mathrm{i}+)$ & $373(32.7)$ & $136(31.2)$ & $190(33.6)$ & $47(33.3)$ \\
\hline pN1 & $612(53.6)$ & $235(53.9)$ & $300(53.1)$ & $77(54.6)$ \\
\hline $\mathrm{pN} 2 / \mathrm{pN} 3$ & $157(13.8)$ & $65(14.9)$ & $75(13.3)$ & $17(12.1)$ \\
\hline \multicolumn{5}{|l|}{ Histological grade-no. (\%) } \\
\hline Grade I & $202(18.2)$ & $72(16.9)$ & $103(18.7)$ & $27(20.0)$ \\
\hline Grade II & $571(51.4)$ & $226(53.1)$ & $277(50.4)$ & $68(50.4)$ \\
\hline Grade III & $338(30.4)$ & $128(30.0)$ & $170(30.9)$ & $40(29.6)$ \\
\hline Unknown & 31 & 10 & 15 & 6 \\
\hline \multicolumn{5}{|l|}{ Hormone receptor status-no. $(\%)$} \\
\hline ER and $P R$ positive & $877(76.8)$ & $346(79.4)$ & $428(75.8)$ & $103(73.1)$ \\
\hline ER or PR positive & $265(23.2)$ & $90(20.6)$ & $137(24.2)$ & $38(26.9)$ \\
\hline \multicolumn{5}{|l|}{ HER2 status-no. (\%) } \\
\hline Positive & $19(1.8)$ & $7(1.7)$ & $11(2.1)$ & $1(0.7)$ \\
\hline Negative & $1063(98.2)$ & $406(98.3)$ & $523(97.9)$ & $134(99.3)$ \\
\hline Unknown & 60 & 23 & 31 & 6 \\
\hline \multicolumn{5}{|l|}{ Histology-no. (\%) } \\
\hline Lobular & $207(18.1)$ & $80(18.4)$ & $100(17.7)$ & $27(19.2)$ \\
\hline Other & $935(81.9)$ & $356(81.7)$ & $465(82.3)$ & $114(80.9)$ \\
\hline \multicolumn{5}{|l|}{ Type of breast surgery-no. (\%) } \\
\hline Breast-conserving surgery & $568(49.7)$ & $214(49.1)$ & $291(51.5)$ & $63(44.7)$ \\
\hline Mastectomy & $574(50.3)$ & $222(50.9)$ & $274(48.5)$ & $78(55.3)$ \\
\hline \multicolumn{5}{|l|}{ Type of axillary surgery-no. (\%) } \\
\hline Sentinel node only & $310(27.1)$ & $129(29.6)$ & $144(25.5)$ & $37(26.2)$ \\
\hline Axillary lymph node dissection only & $298(26.1)$ & $106(24.3)$ & $158(28.0)$ & $34(24.1)$ \\
\hline $\begin{array}{l}\text { Sentinel node plus axillary lymph node dis- } \\
\text { section }\end{array}$ & $517(45.3)$ & $195(44.7)$ & $253(44.8)$ & $69(48.9)$ \\
\hline None & $17(1.5)$ & $6(1.4)$ & $10(1.7)$ & $1(0.7)$ \\
\hline \multicolumn{5}{|l|}{ Radiotherapy-no. (\%) } \\
\hline Local & $320(28.0)$ & $119(27.3)$ & $162(28.6)$ & $39(27.7)$ \\
\hline Regional lymph nodes & $24(2.1)$ & $12(2.8)$ & $9(1.6)$ & $3(2.1)$ \\
\hline Local and regional lymph nodes & $414(36.3)$ & $159(36.5)$ & $201(37.2)$ & $45(31.9)$ \\
\hline None/unknown & $384(33.6)$ & $146(33.5)$ & $184(32.6)$ & $54(38.3)$ \\
\hline \multicolumn{5}{|l|}{ Prior (neo)adjuvant chemotherapy-no. $(\%)^{\mathrm{a}}$} \\
\hline $\begin{array}{l}\text { Anthracycline- and taxane-containing regi- } \\
\text { men }\end{array}$ & $77(6.7)$ & $25(5.7)$ & $39(6.9)$ & $13(9.2)$ \\
\hline $\begin{array}{l}\text { Anthracycline-containing regimen without } \\
\text { taxane }\end{array}$ & $712(62.3)$ & $281(64.4)$ & $350(61.9)$ & $81(57.4)$ \\
\hline $\begin{array}{l}\text { Taxane-containing regimen without anthra- } \\
\text { cycline }\end{array}$ & $6(0.5)$ & $1(0.2)$ & $4(0.7)$ & $1(0.7)$ \\
\hline
\end{tabular}


Table 1 (continued)

\begin{tabular}{lllll}
\hline Characteristic & Total group $(n=1142)$ & Normal BMD $(n=436)$ & Osteopenia $(n=565)$ & Osteoporosis $(n=141)$ \\
\hline $\begin{array}{l}\text { Regimen without anthracycline or taxane } \\
\text { No chemotherapy }\end{array}$ & $19(1.7)$ & $6(1.4)$ & $13(2.3)$ & $0(0.0)$ \\
$\begin{array}{l}\text { Prior HER2-targeted therapy_no. }(\%) \\
\text { Yes }\end{array}$ & $328(28.7)$ & $123(28.2)$ & $159(10.4)$ & $46(32.6)$ \\
$\begin{array}{l}\text { Previous duration of tamoxifen } \\
\text { Median and IQR (years) }\end{array}$ & $3(0.4)$ & $3(1.0)$ & $0(0.0)$ & $0(0.0)$ \\
$\begin{array}{l}\text { Treatment with bone protecting agents at inclusion-no. (\%) } \\
\text { Bisphosphonates }\end{array}$ & $139(12.2)$ & $2.3(2.1-2.5)$ & $2.3(2.1-2.5)$ & $2.3(2.1-2.8)$ \\
Vitamin D and/or Calcium & $375(32.8)$ & $5(1.2)$ & & $73(12.9)$ \\
\hline
\end{tabular}

$T X$ size of tumour could not be assessed, ER: oestrogen receptor, $P R$ progesterone receptor, $H E R 2$ human epidermal growth factor receptor 2

${ }^{a}$ All patients received cyclophosphamide-based chemotherapy

and osteoporosis in 141 (12.3\%) patients. Seventeen (3.4\%) patients of the normal BMD group used bisphosphonates in comparison with $161(28.5 \%)$ in the osteopenia group, and $112(80.9 \%)$ in the osteoporosis group. The median follow-up from the landmark was 5.0 years (interquartile range 4.3 to 5.7). The number of DRFS events were 61 and 66 in the 6-and 3-year arm, respectively. In the 6-year arm the 5-year residual DRFS rate was $89.7 \%$ in the osteopenia group, $86.7 \%$ in the osteoporosis group, and $88.9 \%$ in the normal BMD group [osteopenia versus normal BMD: adjusted HR 0.91 (95\% CI 0.53-1.58); osteoporosis versus normal BMD: adjusted HR 1.40 (95\% CI $0.62-3.17)]$. In the 3-year treatment arm the 5-year residual DRFS rate was $89.2 \%$ in the osteopenia group, $89.7 \%$ in the osteoporosis group and $85.8 \%$ in the normal BMD group [osteopenia versus normal BMD: adjusted HR 0.86 (95\% CI 0.51-1.44); osteoporosis versus normal BMD: adjusted HR 0.85 (95\% CI 0.37-1.94)] (Fig. 1a, b).

When we repeated the analyses selecting only those patients who did not use bisphosphonates $(n=852)$ we neither observed an impact of BMD on late DRFS [6-year arm osteopenia versus normal BMD: adjusted HR 0.82 (95\% CI 0.45-1.49); osteoporosis versus normal BMD: adjusted HR 1.10 (95\% CI 0.26-4.67); 3-year arm osteopenia versus normal BMD: adjusted HR 0.75 (95\% CI 0.40-1.42); osteoporosis versus normal BMD: adjusted HR 1.86 (95\% CI 0.43-8.01)] (Fig. 2a, b). The number of 5-year DRFS events were 42 and 50 in the 6- and 3-year arm, respectively.

Bisphosphonate treatment was started at a median $T$-score of -2.3 (IQR -2.7 to -1.7 ). Of the patients in whom bisphosphonates were prescribed, the majority used oral bisphosphonates $(59.3 \%$ alendronate, $27.0 \%$ risedronate, $0.6 \%$ clodronate, $6.6 \%$ ibandronate) and few used intravenous bisphosphonates (3.2\% pamidronate, $2.7 \%$ zoledronate) [12]. Only $0.6 \%$ received denosumab. After a median follow-up of 5.0 years, the use of bisphosphonates before the landmark did not lead to a better DRFS in each of the BMD categories in comparison with women without bisphosphonates, normal BMD unadjusted HR - 0.95 (95\% CI 0.23-3.88), osteopenia unadjusted HR 1.42 (95\% CI 0.84-2.41), and osteoporosis unadjusted HR 0.78 (95\% CI 0.25-2.43) (Fig. 3a-c).

\section{Discussion}

The phase III DATA study investigates the optimal duration of adjuvant anastrozole (6 versus 3 years) in postmenopausal women with hormone receptor-positive breast cancer, after previous 2-3 years of adjuvant tamoxifen. In this preplanned side-study we found no relationship between BMD and late DRFS (more than 5 years after diagnosis). Moreover, we did not observe a relationship between bisphosphonate treatment, predominantly started for a reduced BMD, and late DRFS.

Multiple observational trials observed that a reduced BMD was associated with a lower risk of developing breast cancer $[10,11]$, but it is insufficiently clear whether a reduced BMD in early breast cancer patients is related to a lower risk of distant recurrences. We were not able to show such a relationship in DATA patients, neither after adjusting for bisphosphonate use, as this could be a potential confounder. To our knowledge, this has only been studied (partly) in four studies. First, in the MA.14 trial it was studied whether baseline beta C-telopeptide, a marker of bone resorption, predicted relapse in postmenopausal breast cancer patients [15]. They showed that a higher bone resorption was associated with a higher incidence of bone metastases [HR 2.80 (95\% CI $1.05-7.48 ; p 0.03)]$ during follow-up. Adjustment for bisphosphonate use was not performed, patients were treated with tamoxifen instead of an aromatase inhibitor, and importantly, no information on BMD measurements was available [15]. Also a sub-study of the AZURE trial found the bone turnover markers P1NP, CTX, and 
Fig. 1 The impact of BMD on distant recurrence-free survival for the patients in a the 3-year anastrozole treatment arm, b the 6-year anastrozole treatment arm. Hazard ratios were adjusted for tumour size, nodal status, tumour grade and hormone receptor status
A

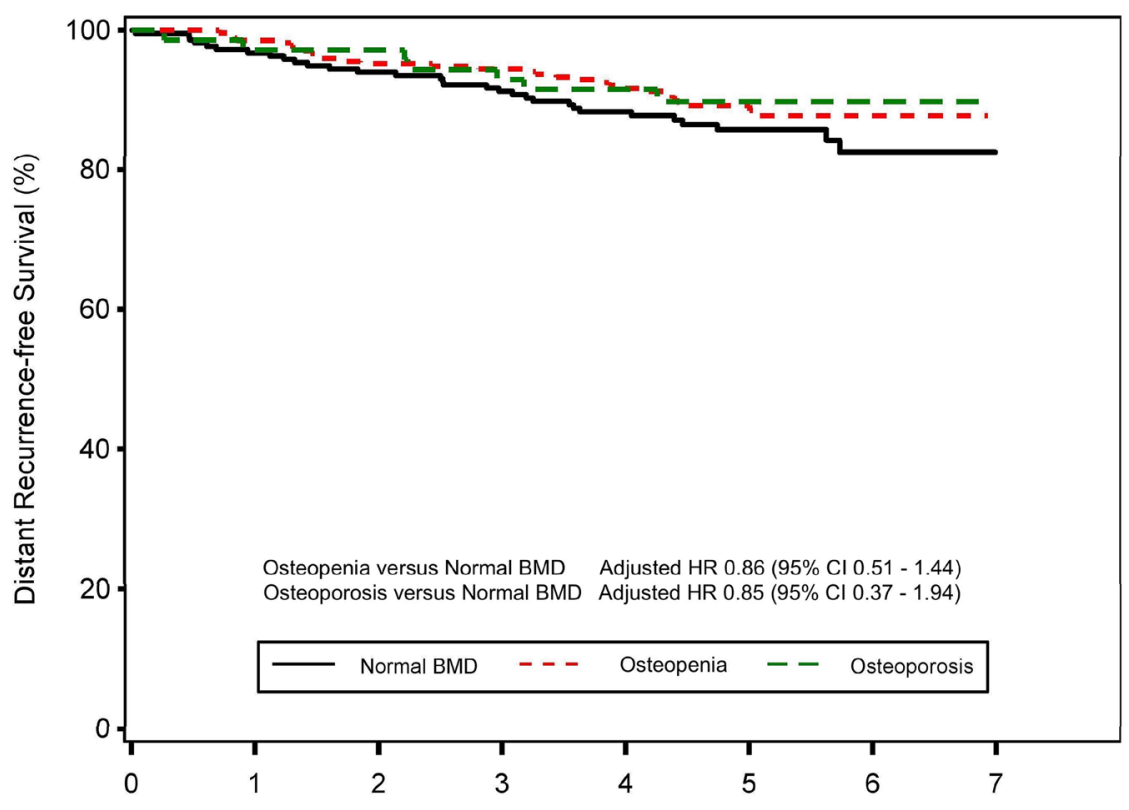

Number at Risk Normal BMD Osteopenia Osteoporosis
Follow-up from landmark 3 year (years)

$\begin{array}{cccccccc}217 & 210 & 204 & 195 & 165 & 100 & 36 & 9 \\ 273 & 268 & 259 & 254 & 209 & 131 & 36 & 4 \\ 72 & 69 & 69 & 65 & 57 & 32 & 13 & 2\end{array}$

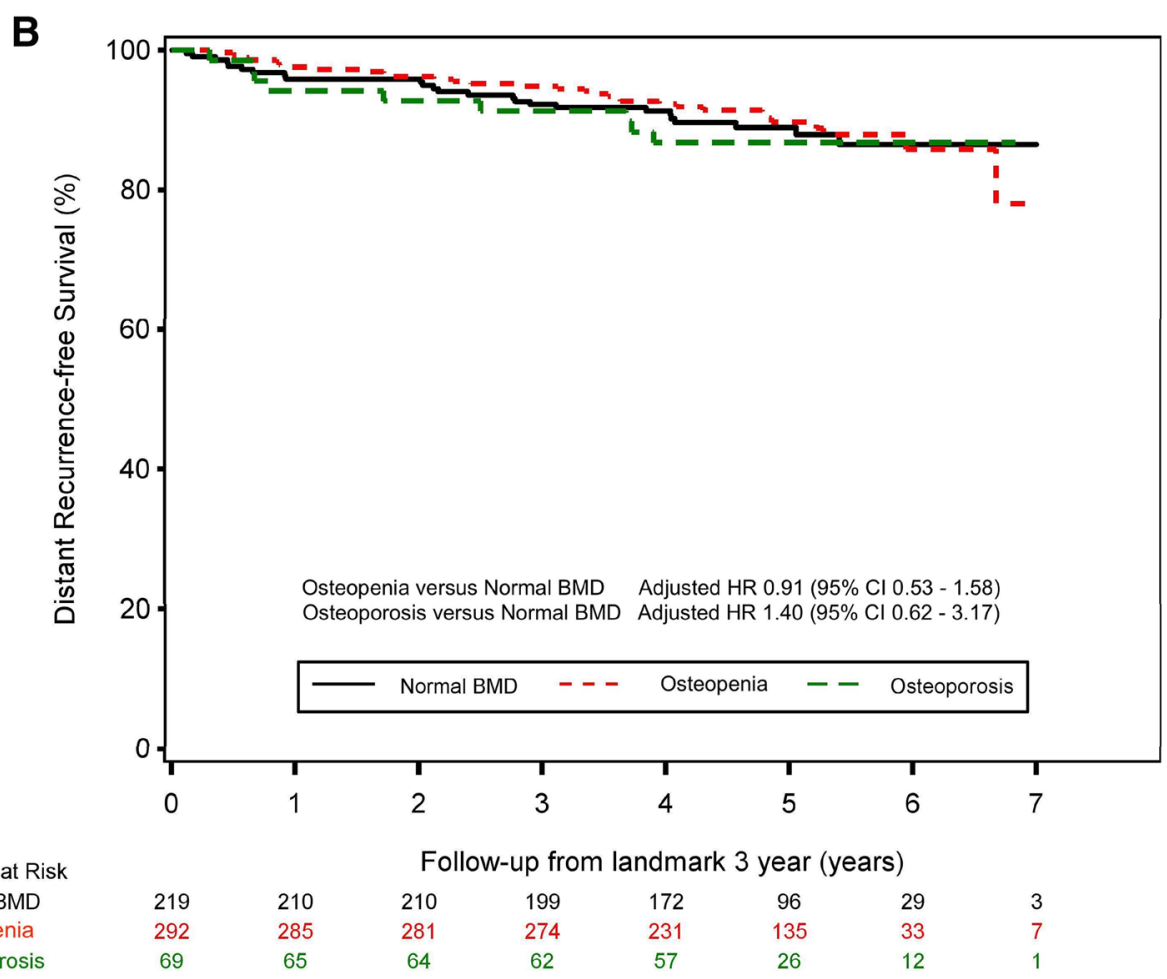

1-CTP to show good prognostic ability for bone-specific recurrence [16]. None of the markers were prognostic for overall distant recurrence and not predictive of treatment benefit from zoledronic acid. A cohort study showed that pre- breast cancer osteoporosis was not associated with risk of developing bone metastasis [17]. Nevertheless, if patients with untreated pre-cancer osteoporosis developed bone metastases, it occurred approximately 1 year earlier than those without pre-cancer osteoporosis (median time, 1.78 years vs 2.87 years; $p<0.001)$. The fourth study, the 
Fig. 2 The impact of BMD on late DRFS selecting only the patients without bisphosphonates before the landmark in a the 6-year anastrozole treatment arm, $\mathbf{b}$ the 3-year anastrozole treatment arm. Hazard ratios were adjusted for tumour size, nodal status, tumour grade and hormone receptor status

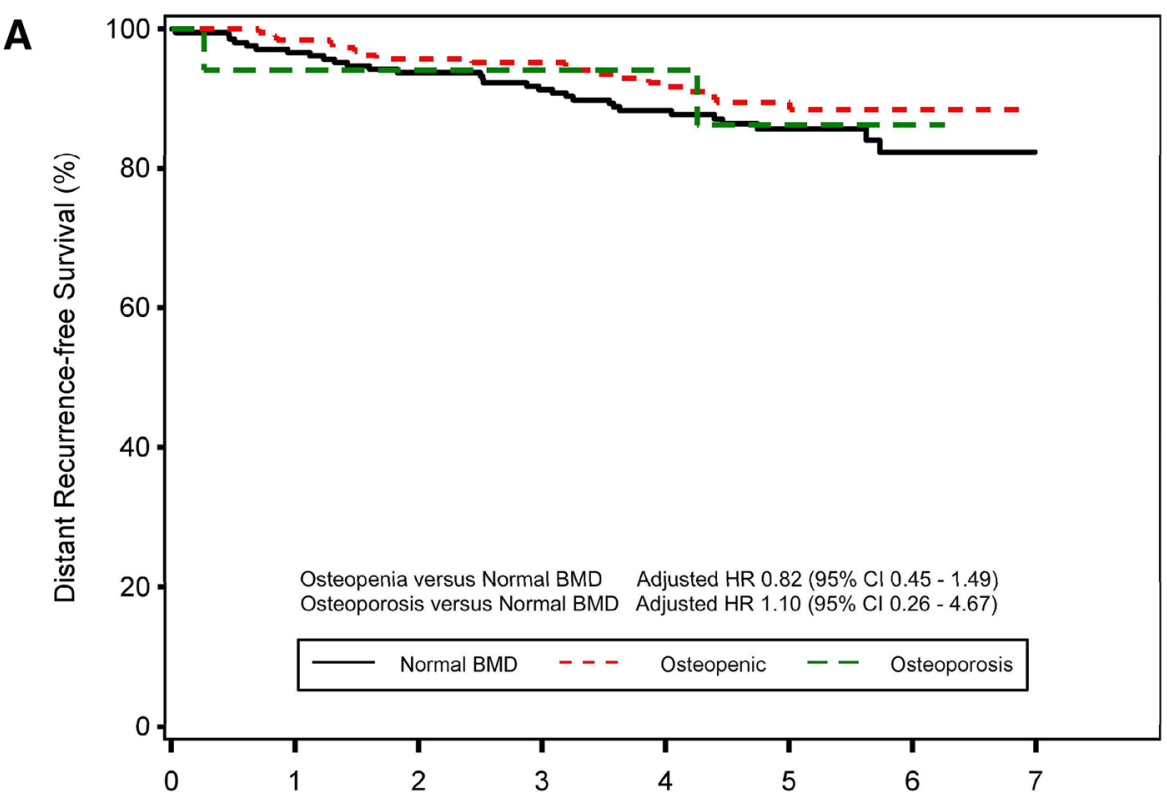

\begin{tabular}{lcccccccc} 
Number at Risk & \multicolumn{7}{c}{ Follow-up from landmark 3 year (years) } \\
Normal BMD & 209 & 202 & 196 & 189 & 160 & 96 & 36 & 9 \\
Osteopenic & 189 & 186 & 181 & 178 & 142 & 89 & 26 & 1 \\
Osteoporosis & 17 & 16 & 16 & 16 & 13 & 7 & 3 & 0
\end{tabular}

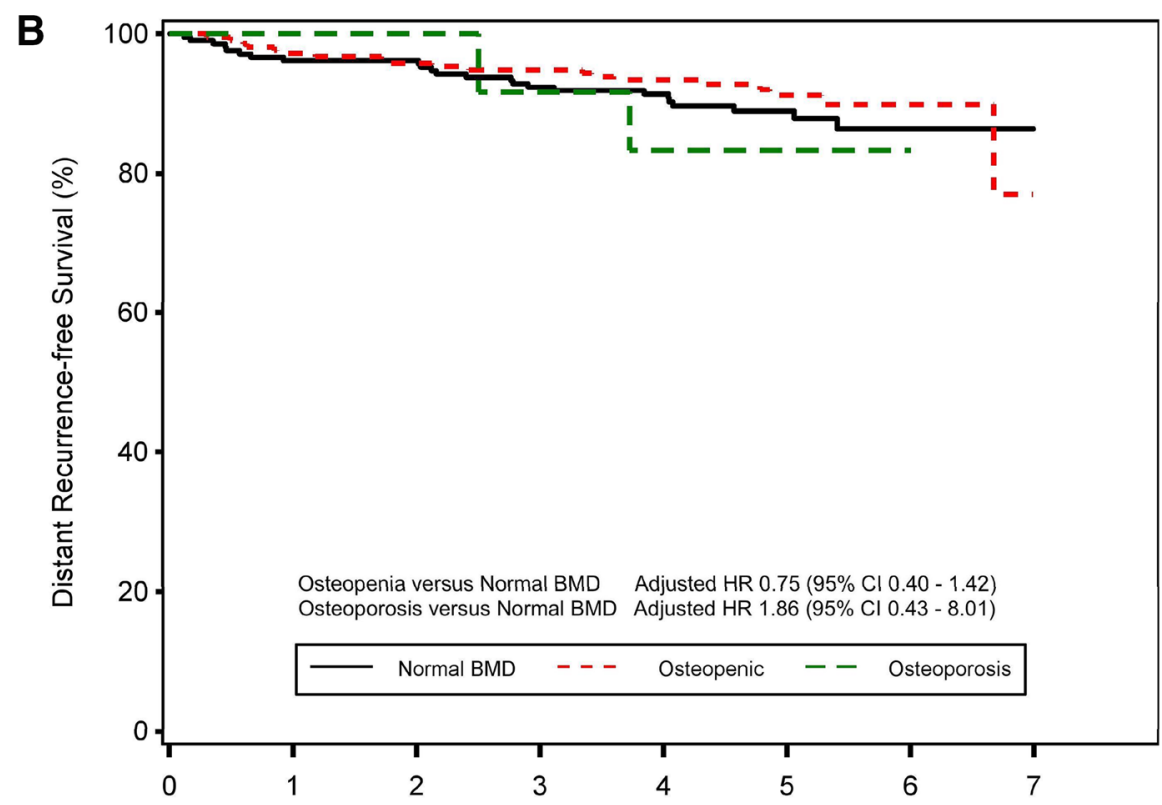

Number at Risk Normal BMD Osteopenic Osteoporosis

Follow-up from landmark 3 year (years)

\begin{tabular}{cccccccc}
\multicolumn{8}{c}{ Follow-up from } \\
210 & 202 & 202 & 191 & 165 & 92 & 28 & 3 \\
215 & 209 & 206 & 201 & 173 & 99 & 24 & 4 \\
12 & 12 & 12 & 11 & 10 & 7 & 0 & 0
\end{tabular}

MA.27 trial, examined the effects of self-reported osteoporosis and osteoporosis therapy on breast cancer outcomes. The study included 7576 postmenopausal patients during adjuvant aromatase inhibitor treatment (anastrozole/exemestane) for breast cancer [18]. Of patients who did not receive bisphosphonates, the event-free survival rate was $86 \%$ (95\% CI 78-91\%) in case of osteoporosis $(n=193)$ and $87 \%$ (95\% CI 86-89\%) in patients without osteoporosis $(n=4672)$ (no HR reported). While their findings are in line with the results of the current study, it 
Fig. 3 The impact of bisphosphonate use before the landmark on late DRFS in the women with A) a normal BMD, B) osteopenia, and C) osteoporosis at the 3-year landmark

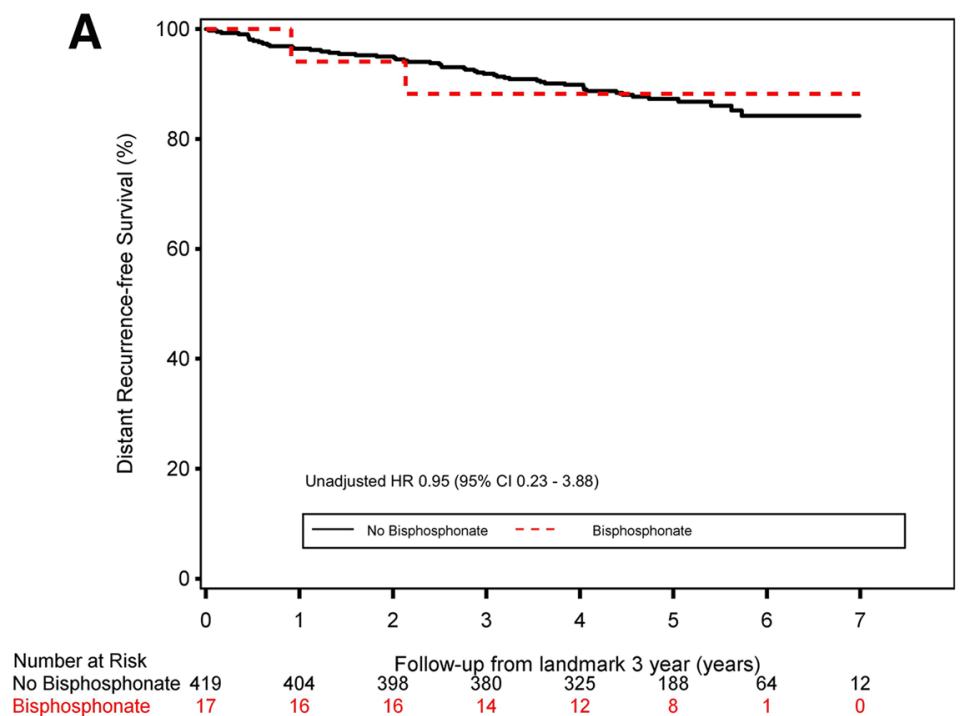

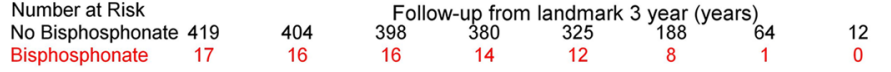

B

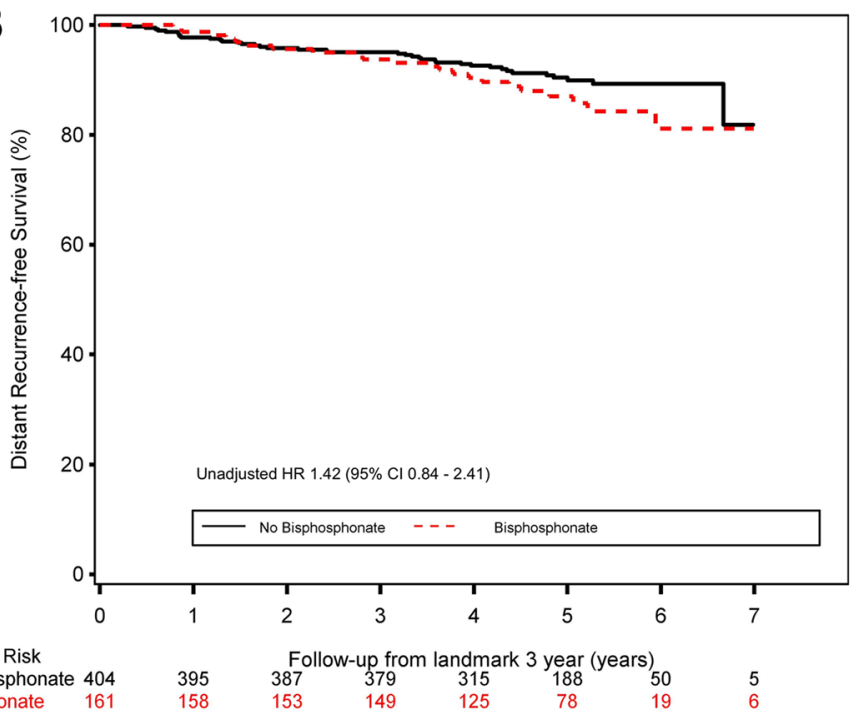

Number at Risk $\begin{array}{lllllllll}\text { No Bisphosphonate } & 404 & 395 & 387 & 379 & 315 & 188 & 50 & 5 \\ \text { Bisphosphonate } & 161 & 158 & 153 & 149 & 125 & 78 & 19 & 6\end{array}$

\section{C}

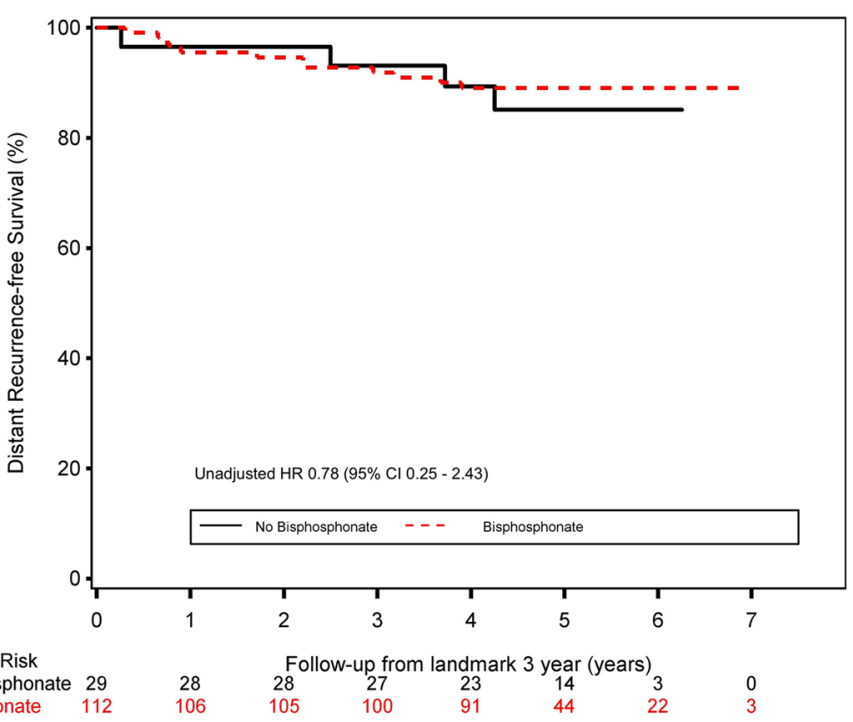


should be recognised that in the MA.27 trial no detailed information was collected on BMD assessments and information on osteoporosis was self-reported [18]. Therefore, current evidence suggests that bone resorption markers might be much more valuable in predicting bone-specific recurrences than BMD.

A possible explanation for not finding an association between a reduced BMD and a lower breast cancer recurrence risk might be that women with osteoporosis have less benefit of aromatase inhibitors because their oestrogen levels tend to be lower than in women with a normal BMD. Hence, in patients with lower intrinsic oestrogen levelsresulting in higher risk of osteoporosis-the breast recurrence risk is reduced in a similar way as in patients with intrinsic higher oestrogen levels treated with aromatase inhibitors.

Earlier studies found bisphosphonates to be valuable in both breast cancer prevention and improved breast cancer survival (when given as adjuvant therapy) irrespective of bisphosphonate type [1-3, 7, 19]. Further, another trial showed that cessation of bisphosphonate treatment after breast cancer diagnosis doubled the risk of developing bone metastases ( $\mathrm{HR}=2.03,95 \%$ CI 1.26 to 3.26 ), whereas taking bisphosphonates post-breast cancer diagnosis only, or continuing post-diagnosis reduced the risk of bone metastases (45\% and $28 \%$ relative reduction, respectively) after a median 5-year follow-up [20]. Also in the MA.27 trial a 5 -year absolute $3 \%$ improvement of the event-free survival was observed for the patients receiving osteoporosis therapy in comparison with the patients who did not receive osteoporosis therapy [ $86 \%$ versus $89 \%$, HR 0.63 (95\% CI 0.40-1.00)] during adjuvant aromatase inhibitor therapy for breast cancer [18]. The benefit was larger in patients without osteoporosis [ $87 \%$ versus $92 \%$, HR 0.65 (95\% CI $0.61-0.68)]$. The bisphosphonate treatment in our study was also predominantly started for a reduced BMD; however, we did not observe an effect on DRFS. A possible explanation for the diverging observations is that in the MA.27 trial a standard Cox regression analysis was used, potentially overestimating the treatment effect of osteoporosis therapy by introducing 'immortal time bias' [21]. When the start of osteoporosis therapy was used as a time dependent covariate the effect on DRFS was not found [18]. Therefore it could be possible that adjuvant bisphosphonate treatment is most effective in patients without osteoporosis. Another explanation for not finding a relationship between bisphosphonate use and late DRFS is the use of the oral bisphosphonates alendronate and risedronate in $86 \%$ of the patients using bisphosphonates [12]. The EBCTCG meta-analysis on adjuvant use of bisphosphonates concluded there was insufficient evidence for the use of alendronate and risedronate in the adjuvant setting of breast cancer [7]. Nevertheless, non-prospective trials suggest this relationship also exists for these oral bisphosphonates [22, 23]. Hopefully, future prospective trials will clarify this matter.

Bisphosphonates inhibit osteoclastic bone resorption by attaching to bony surfaces undergoing active resorption and prevent osteocyte and osteoblast apoptosis [24, 25]. Through these mechanisms bisphosphonates increase the BMD, decrease the incidence of osteoporotic fractures, and were implemented as therapy for metastatic skeletal disease [26]. However, several in vitro studies found that bisphosphonates might not only target the osteoclast, but also have direct anti-tumour activity and work synergistic with cytotoxic therapies [27-29]. These might be the mechanisms responsible for the observed clinical benefits in trials investigating the efficacy of zoledronic acid in combination with standard anticancer therapy in early breast cancer patients $[2$, $4,5]$. Noticeably the indirect metastasis-preventing effect of bisphosphonates seems limited to postmenopausal patients [7], which implies that the effect of oestrogen on the bone microenvironment might play an important role in the benefit from adjuvant bisphosphonate therapy [30].

More recently, the effect of the anti-receptor activator of nuclear factor kappa-B ligand denosumab on breast cancer survival was investigated, showing contrasting results [31, 32]. The ABCSG-18 trial observed a clear advantage of denosumab (60 mg 6-monthly) on disease-free survival (DFS) (HR 0.82, $p=0.026$ ) in a study population of postmenopausal women using AIs, who were generally at a low risk of recurrence ( $25 \%$ received prior chemotherapy) [32]. In the denosumab group, DFS was $80.6 \%$ at 8 years of follow-up, compared with $77.5 \%$ in the placebo group. The D-CARE trial, including both pre- and postmenopausal women of whom $96 \%$ received prior chemotherapy, observed no advantage of a more intense regimen of adjuvant denosumab (120 mg monthly for 6 months followed by $120 \mathrm{mg}$ 3-monthly) on DFS (HR 1.04, $p=0.57$ ) [31]. A subgroup analysis neither showed an effect for the subgroup of postmenopausal women [31]. Because of these contrasting results denosumab has not been registered (yet) as adjuvant treatment in women with breast cancer. In terms of adverse events, osteonecrosis in the jaw was reported significantly more often in the denosumab arm in the D-CARE trial as compared to placebo [31]. The ABCSG-18 trial, using a lower dose of denosumab, did not observe any differences in the occurrence of osteonecrosis between the use of adjuvant denosumab and placebo [32]. Further, the Medicines and Healthcare products Regulatory Agency recently cautioned that denosumab has been associated with an increased incidence of new primary malignancies (1-year cumulative incidence $1.1 \%$ ) [33].

Even though this was a planned side-study of the DATA trial, the execution of DEXA scans was not protocolized but was advised to adhere to (inter)national guidelines. This probably explains the absence of BMD measurements within 
3 years after randomisation in about one third of the patients. Furthermore, $19.1 \%$ of the women with osteoporosis did not use any bisphosphonates which was not in accordance with the recommendations in the national guideline. Additionally, the use of bisphosphonates was not randomised but based on the outcome of the DEXA scans, therefore confounding by indication could not be ruled out. The occurrence of bone metastases was not registered as specific item anymore after the occurrence of distant metastases elsewhere, and therefore we could not use bone metastases free survival as an outcome. Also, with longer follow-up results may change. Nevertheless, this is the first prospective trial studying the relationship between BMD and DRFS with detailed information on BMD in 1142 patients.

In conclusion, we observed no association between BMD and late DRFS in this pre-planned DATA sub-study. Neither did we observe a relationship between bisphosphonate use for a decreased BMD and late DRFS.

Acknowledgements We thank Wim A.J.G. Lemmens, Bianca Schalk, and Ton de Haan for their assistance with performing the statistical analyses.

Funding This study was funded by AstraZeneca NL, ClinicalTrials. gov number, NCT00301457.

\section{Compliance with ethical standards}

Conflicts of interest IEGvH, SCL and ACPS report institutional grants from AstraZeneca during the conduct of the study. MdB reports research grants from Roche, Eisai, Novartis, Pfizer, and Lily during the conduct of the study; and consultation/speakers fee from Roche, Novartis, and Pfizer. VCGT-H reports consultant/advisory role to Pfizer, Lily, and Novartis, and grants to the institute for research from AstraZeneca, Roche, Novartis, Pfizer, and Lilly during the conduct of the study. FWPJvdB, FLGE, HdG, AH, ALTI, JRK, PGMP, WKdR, MJCvdS, CMS, CHS declare no conflicts of interests.

Ethical approval All procedures performed in studies involving human participants were in accordance with the ethical standards of the institutional and/or national research committee and with the 1964 Helsinki declaration and its later amendments or comparable ethical standards. This article does not contain any studies with animals performed by any of the authors.

Informed consent Informed consent was obtained from all individual participants included in the study.

Open Access This article is licensed under a Creative Commons Attribution 4.0 International License, which permits use, sharing, adaptation, distribution and reproduction in any medium or format, as long as you give appropriate credit to the original author(s) and the source, provide a link to the Creative Commons licence, and indicate if changes were made. The images or other third party material in this article are included in the article's Creative Commons licence, unless indicated otherwise in a credit line to the material. If material is not included in the article's Creative Commons licence and your intended use is not permitted by statutory regulation or exceeds the permitted use, you will need to obtain permission directly from the copyright holder. To view a copy of this licence, visit http://creativecommons.org/licenses/by/4.0/.

\section{References}

1. Paterson AH, Anderson SJ, Lembersky BC et al (2012) Oral clodronate for adjuvant treatment of operable breast cancer (National Surgical Adjuvant Breast and Bowel Project protocol B-34): a multicentre, placebo-controlled, randomised trial. Lancet Oncol 13:734-742

2. Coleman R, Cameron D, Dodwell D et al (2014) Adjuvant zoledronic acid in patients with early breast cancer: final efficacy analysis of the AZURE (BIG 01/04) randomised open-label phase 3 trial. Lancet Oncol 15:997-1006

3. Powles T, Paterson S, Kanis JA et al (2002) Randomized, placebocontrolled trial of clodronate in patients with primary operable breast cancer. J Clin Oncol 20:3219-3224

4. Gnant M, Mlineritsch B, Stoeger H et al (2011) Adjuvant endocrine therapy plus zoledronic acid in premenopausal women with early-stage breast cancer: 62-month follow-up from the ABCSG12 randomised trial. Lancet Oncol 12:631-641

5. Coleman R, de Boer R, Eidtmann H et al (2013) Zoledronic acid (zoledronate) for postmenopausal women with early breast cancer receiving adjuvant letrozole (ZO-FAST study): final 60-month results. Ann Oncol 24:398-405

6. Brufsky AM, Harker WG, Beck JT et al (2012) Final 5-year results of Z-FAST trial: adjuvant zoledronic acid maintains bone mass in postmenopausal breast cancer patients receiving letrozole. Cancer 118:1192-1201

7. Early Breast Cancer Trialists' Collaborative Group (2015) Adjuvant bisphosphonate treatment in early breast cancer: meta-analyses of individual patient data from randomised trials. Lancet 386:1353-1361

8. Chlebowski RT, Chen Z, Cauley JA et al (2010) Oral bisphosphonate use and breast cancer incidence in postmenopausal women. $\mathrm{J}$ Clin Oncol 28:3582-3590

9. Kroep JR, Charehbili A, Coleman RE et al (2016) Effects of neoadjuvant chemotherapy with or without zoledronic acid on pathological response: A meta-analysis of randomised trials. Eur J Cancer 54:57-63

10. Zhang Y, Kiel DP, Kreger BE et al (1997) Bone mass and the risk of breast cancer among postmenopausal women. N Engl J Med 336:611-617

11. Zmuda JM, Cauley JA, Ljung BM, Bauer DC, Cummings SR, Kuller LH (2001) Bone mass and breast cancer risk in older women: differences by stage at diagnosis. J Natl Cancer Inst 93:930-936

12. van Hellemond IEG, Smorenburg CH, Peer PGM et al (2019) Assessment and management of bone health in women with early breast cancer receiving endocrine treatment in the DATA study. Int J Cancer 145:1325-1333

13. Tjan-Heijnen VCG, van Hellemond IEG, Peer PGM et al (2017) Extended adjuvant aromatase inhibition after sequential endocrine therapy (DATA): a randomised, phase 3 trial. Lancet Oncol 18:1502-1511

14. WHO Scientific Group on the Assessment of Osteoporosis at Primary Health Care Level (2007) Summary Meeting Report. World Health Organization. https://www.who.int/chp/topics/Osteoporos is.pdf. Accessed 23 Dec 2015

15. Lipton A, Chapman JA, Demers L et al (2011) Elevated bone turnover predicts for bone metastasis in postmenopausal breast cancer: results of NCIC CTG MA.14. J Clin Oncol 29:3605-3610 
16. Brown J, Rathbone E, Hinsley S et al (2018) Associations between serum bone biomarkers in early breast cancer and development of bone metastasis: results from the AZURE (BIG01/04) trial. J Natl Cancer Inst (JNCI) 110:871-879

17. Chen HM, Chen FP, Yang KC, Yuan SS (2019) Association of bone metastasis with early-stage breast cancer in women with and without precancer osteoporosis according to osteoporosis therapy status. JAMA Netw Open 2:e190429

18. Lipton A, Chapman JW, Leitzel K et al (2017) Osteoporosis therapy and outcomes for postmenopausal patients with hormone receptor-positive breast cancer: NCIC CTG MA.27. Cancer 123:2444-2451

19. Bouvard B, Chatelais J, Soulie P et al (2018) Osteoporosis treatment and 10 years' oestrogen receptor+ breast cancer outcome in postmenopausal women treated with aromatase inhibitors. Eur J Cancer 101:87-94

20. Kremer R, Gagnon B, Meguerditchian AN, Nadeau L, Mayo N (2014) Effect of oral bisphosphonates for osteoporosis on development of skeletal metastases in women with breast cancer: results from a pharmaco-epidemiological study. J Natl Cancer Inst. https ://doi.org/10.1093/jnci/dju264

21. Agarwal P, Moshier E, Ru M et al (2018) Immortal time bias in observational studies of time-to-event outcomes: assessing effects of postmastectomy radiation therapy using the national cancer database. Cancer Control 25:1073274818789355

22. Rennert G, Pinchev M, Gronich N et al (2017) Oral bisphosphonates and improved survival of breast cancer. Clin Cancer Res 23:1684-1689

23. Rouach V, Goldshtein I, Wolf I et al (2018) Exposure to alendronate is associated with a lower risk of bone metastases in osteoporotic women with early breast cancer. J Bone Oncol 12:91-95

24. Rodan GA, Fleisch HA (1996) Bisphosphonates: mechanisms of action. J Clin Invest 97:2692-2696

25. Hughes DE, Wright KR, Uy HL et al (1995) Bisphosphonates promote apoptosis in murine osteoclasts in vitro and in vivo. $\mathrm{J}$ Bone Miner Res 10:1478-1487
26. Hortobagyi GN, Theriault RL, Porter L et al (1996) Efficacy of pamidronate in reducing skeletal complications in patients with breast cancer and lytic bone metastases. Protocol 19 Aredia Breast Cancer Study Group. N Engl J Med 335:1785-1791

27. Solomayer EF, Gebauer G, Hirnle P et al (2012) Influence of zoledronic acid on disseminated tumor cells in primary breast cancer patients. Ann Oncol 23:2271-2277

28. Neville-Webbe HL, Evans CA, Coleman RE, Holen I (2006) Mechanisms of the synergistic interaction between the bisphosphonate zoledronic acid and the chemotherapy agent paclitaxel in breast cancer cells in vitro. Tumor Biol 27:92-103

29. Neville-Webbe HL, Coleman RE, Holen I (2010) Combined effects of the bisphosphonate, zoledronic acid and the aromatase inhibitor letrozole on breast cancer cells in vitro: evidence of synergistic interaction. Br J Cancer 102:1010-1017

30. Ottewell PD, Wang N, Brown HK et al (2014) Zoledronic acid has differential antitumor activity in the pre- and postmenopausal bone microenvironment in vivo. Clin Cancer Res 20:2922-2932

31. Coleman RE, Finkelstein D, Barrios CH et al (2018) Adjuvant denosumab in early breast cancer: first results from the international multicenter randomized phase III placebo controlled D-CARE study. J Clin Oncol 36:501

32. Gnant M, Pfeiler G, Steger GG et al (2019) Adjuvant denosumab in postmenopausal patients with hormone receptor-positive breast cancer (ABCSG-18): disease-free survival results from a randomised, double-blind, placebo-controlled, phase 3 trial. Lancet Oncol 20:339-351

33. Medicines and Healthcare products Regulatory Agency (2018) Denosumab (Xgeva) for advanced malignancies involving bone: study data show new primary malignancies reported more frequently compared to zoledronate. Drug Saf Update 11(11):3

Publisher's Note Springer Nature remains neutral with regard to jurisdictional claims in published maps and institutional affiliations.

\section{Affiliations}

\section{Irene E. G. van Hellemond ${ }^{1}$. Carolien H. Smorenburg ${ }^{2}$ - Petronella G. M. Peer ${ }^{3}$. Astrid C. P. Swinkels ${ }^{4}$. Caroline M. Seynaeve ${ }^{5}$. Maurice J. C. van der Sangen ${ }^{6}$. Judith R. Kroep ${ }^{7}$. Hiltje de Graaf ${ }^{8}$. Aafke H. Honkoop ${ }^{9}$. Frans L. G. Erdkamp ${ }^{10}$. Franchette W. P. J. van den Berkmortel ${ }^{11}$. Wilfred K. de Roos ${ }^{12}$. Sabine C. Linn ${ }^{13}$. Alexander L. T. Imholz ${ }^{14}$. Maaike de Boer ${ }^{1}$. Vivianne C. G. Tjan-Heijnen ${ }^{1,15}$ on behalf of the Dutch Breast Cancer Research Group (BOOG)}

1 Department of Medical Oncology, GROW - School for Oncology and Developmental Biology, Maastricht University Medical Centre, Maastricht, The Netherlands

2 Department of Internal Medicine, Medical Centre Alkmaar, Alkmaar, The Netherlands

3 Biostatistics, Radboud Institute for Health Sciences, Radboud University Medical Centre, Nijmegen, The Netherlands

4 Clinical Research Department, Netherlands Comprehensive Cancer Organization IKNL, Utrecht, The Netherlands

5 Department of Medical Oncology, Erasmus MC Cancer Institute, Rotterdam, The Netherlands

6 Department of Radiation Oncology, Catharina Hospital, Eindhoven, The Netherlands
7 Department of Medical Oncology, Leiden University Medical Centre, Leiden, The Netherlands

8 Department of Medical Oncology, Medical Centre Leeuwarden, Leeuwarden, The Netherlands

9 Department of Medical Oncology, Isala Clinics, Zwolle, The Netherlands

10 Department of Medical Oncology, Zuyderland Medical Centre, Sittard, The Netherlands

11 Department of Medical Oncology, Zuyderland Medical Centre, Heerlen, The Netherlands

12 Department of Surgery, Gelderse Vallei Hospital, Ede, The Netherlands 
13 Department of Medical Oncology, Netherlands Cancer Institute, Amsterdam, The Netherlands

14 Department of Medical Oncology, Deventer Hospital, Deventer, The Netherlands
15 Division of Medical Oncology, Department of Internal Medicine, Maastricht University Medical Centre,

P.O. Box 5800, 6202 AZ Maastricht, The Netherlands 